\title{
Die Expertengruppen der EU-Kommission und das Paradigma der Brüsseler Technokratie
}

\author{
Julia Metz
}

\begin{abstract}
Kernaussagen
Der Politikprozess in der Europäischen Union ist zunehmend von Experten dominiert. Im Zentrum steht die EUKommission, die aufgrund ihres Personalmangels häufig auf externe Sachverständige zurückgreift. So gilt das enge Zusammenspiel zwischen EU-Kommission und Experten als Sinnbild technokratischen Regierens. Dieser Beitrag argumentiert, dass diese Sichtweise verkennt, dass die Einbindung technokratischer Institutionen nicht zwangsläufig einen technokratischen motivierten Politikprozess bedingt. Eine quantitative und qualitative Analyse zeigt, dass die EUKommission Expertengruppen nicht nur technokratisch, zur Wissensbeschaffung, sondern auch politisch, zur Legitimation und zur Konsensbildung, nutzt. Unter dem Deckmantel der Brüsseler Technokratie verbergen sich daher oft auch politische Prozesse.
\end{abstract}

\section{Einleitung ${ }^{1}$}

In Zeiten wirtschaftlicher und politischer Krisen greifen Politiker verstärkt auf externe Sachverständige zurück, die Unsicherheit reduzieren und konkrete Handlungsanweisungen liefern sollen (Haas, 1992). Die aktuelle Eurokrise ist ein gutes Beispiel für ein komplexes, vielschichtiges Problem, dessen Interdependenz mit den schnelllebigen Finanzmärkten rasche politische Handlungsanweisungen verlangt. Bei der Erarbeitung von Lösungsvorschlägen rückt die Expertise externer Sachverständige in den Mittelpunkt. In Griechenland und Italien wurde diese „Technokratisierung“ der Politik jüngst bis zum Äußersten getrieben, indem zur Krisenbewältigung die gewählten Regierungen zeitweilig durch ungewählte, technokratische Wirtschaftsexperten ersetzt wurden (Kaube, 2012; Streeck, 2012). Weniger drastisch, aber dennoch deutlich, schildert der Finanzexperte der SPD-Bundestagsfraktion Carsten Schneider in einem Interview mit der Wochenzeitung Die ZEIT die Abhängigkeit der Politik von Technokraten. Während er die Einbindung externer Experten durchaus als „Gefahr für die Demokratie“ erkenne, fehle Politikern in Zeiten der „Hektik“ jedoch „schlicht die Zeit [...sich...] in alles zu vertiefen“ (Bittner, 2012). Der notwendige Rückgriff auf externes Fachwissen führe zu einer kontinuierlichen Interaktion mit Wissenschaftlern und Praktikern aus der Finanzwirtschaft und führe zu einer Abhängigkeit der Politiker von externen Sachverständigen: „Ich kann und muss nicht alle Details über die Eurokrise wissen - aber mich auf Leute verlassen können, deren Wissen ich vertraue“ (Bittner, 2012).
Wie vom Parlamentarier erkannt, berührt die Einbindung von Experten in die Politikgestaltung den Grundkonflikt zwischen Technokratie und Demokratie. Während in einer Technokratie Expertenwissen als Macht- und Autoritätsbasis dient, beruht die Demokratie auf dem Prinzip bürgerlicher Partizipation, wörtlich: der Herrschaft des Volkes. Die Einbindung von Technokraten, die über keinen Wählerauftrag verfügen, mag bei der Lösung komplexer Sachverhalte zwar sachdienlich sein, ist demokratietheoretisch jedoch bedenklich (Radaelli, 1999; Schrage, 2012).

Bei der Eurokrise stellt sich dieser Konflikt verschärft dar, da die Problemlage größtenteils politische Maßnahmen auf der supranationalen Ebene der Europäischen Union (EU) erfordert (Walter, 2012). Die EU leidet jedoch strukturell an einem „Demokratiedefizit“ (Follesdal \& Hix, 2006), ${ }^{2}$ denn die dort getroffenen weitreichenden Entscheidungen sind nicht ausreichend durch demokratische Entscheidungsprozesse legitimiert (der EU mangelt es an „Input-Legitimität“, so Scharpf, 1999). Seine Legitimität erlangt das EU-System vielmehr durch die Effektivität seiner Entscheidungen („,Output-Legitimität“). Doch im Rahmen der aktuellen Eurokrise fehlt es auch diesen vermehrt an Akzeptanz: Die getroffenen Maßnahmen zur Krisenbewältigung lösen sowohl in den adressierten Ländern, wie Griechenland, Spanien oder jüngst Zypern, als auch bei den Geber-Ländern, wie Deutschland oder den Niederlanden, teilweise massiven Widerstand aus (Scharpf, 2012). Das Ergebnis ist ein grassierender Euroskeptizismus (Hartleb, 2011), ${ }^{3}$ der das Schwinden an genereller Akzeptanz für das politische System der EU offenbart, und somit das Legitimitätsproblem der EU verstärkt.

Im Zentrum der Kritik steht dabei häufig die EU-Kommission, die trotz ihrer maßgeblichen Zuständigkeit für die Vorbereitung und Durchführung von EU-Recht über kein demokratisches Mandat verfügt (Tsakatika, 2005). Sie wird daher oft als das Sinnbild einer technokratischen Institution gesehen: „Die EU-Kommission [...] dient vielen als weiterer und eigentlicher Hinweis auf die Dominanz technokratischer Politik heute." (Kaube, 2012: 857). Hinzu kommt, dass die EU-Kommission bei der Bewältigung ihrer Aufgaben stark auf externe

1 Ich bedanke mich bei meinen Kollegen Miriam Hartlapp und Christian Rauh für ihre Hilfe bei der qualitativen Datenerhebung und den anonymen Gutachtern für konstruktive Kritik.

2 Diese Feststellung trifft dann zu, wenn die EU an den Maßstäben nationalstaatlicher repräsentativer Demokratien gemessen wird.

3 Jüngst hat sich dieser in Deutschland in der Bildung der Protestpartei „Alternative für Deutschland“ offenbart, die den undemokratischen Prozess der Euro-Rettung anprangert und die Auflösung des EuroRaums zum Ziel hat. 
Sachverständige zurückgreift, was den „Technokratieüberschuss“ (Kaube, 2012: 857) Brüssels weiter verschärft.

Dieser Beitrag greift den Vorwurf einer Brüsseler Technokratie auf und untersucht inwiefern die unzähligen beratenden Expertengruppen der EU-Kommission Ausdruck technokratischen Regierens sind. Er unterscheidet zwischen technokratischen Institutionen und Politikprozessen und argumentiert, dass Expertengruppen einerseits von ihrer institutionellen Verfasstheit technokratisch sind. Anderseits muss ihre Einbindung bei der Gesetzgebung nicht zwangsläufig auf einen technokratischen, sachorientierten Politikprozess hinweisen. Denn die EU-Kommission nutzt Expertengruppen auch als politisches Instrument zur Legitimationsbeschaffung und Konsensfindung im europäischen Politikprozess. Unter dem Deckmantel der Technokratie kann der Brüsseler Politikprozess also hochpolitisch sein.

Im Folgenden wird zunächst diese Annahme konzeptionell spezifiziert und drei Formen der Expertengruppennutzung eingeführt (Kapitel zwei). Empirisch wird erst die zahlenmäßige Entwicklung der Expertengruppen über Zeit und ihre Verteilung über Politikressorts untersucht, was ambivalente Evidenz für eine technokratische Nutzung liefert (Kapitel drei). Eine qualitative Untersuchung der Funktion der Expertengruppen im politischen Gesetzgebungsprozess zeigt, dass die EU-Kommission Expertengruppen sowohl technokratisch als auch politisch nutzt (Kapitel vier). Kapitel fünf diskutiert die Erkenntnisse der Arbeit im Lichte aktueller Debatten. Die Konklusion fasst die Ergebnisse zusammen.

\section{Vom technischen und politischen Nutzen Sachverständiger}

Die EU-Kommission ist durch ihren institutionellen Aufbau und das ihr gegebene Mandat eine technokratische Institution par excellence. In den 1950er Jahren wurde sie von Jean Monnet als ein von den Mitgliedstaaten unabhängiger, schlanker Expertenstab ins Leben gerufen, dessen Legitimität sich aus seinem unparteiischen und technokratischen Sachverstand speist: Nicht Politiker, sondern eine technokratische Elite sollte den Europäischen Integrationsprozess anleiten (Tsakatika, 2005). Obwohl fachliche Expertise die zentrale Ressource und Legitimation der EU-Kommission ist, verfügt sie im Vergleich zu nationalen Exekutiven nur über unzureichend interne Experten. Allein strukturell ist sie daher auf externes Fachwissen angewiesen, um ihren Aufgaben nachzukommen. So wird sie in ihrer täglichen Arbeit von annähernd 1000 Expertengruppen beraten, die sich aus Interessengruppenvertretern, nationalen Ministerialbeamten oder Wissenschaftlern zusammensetzen, und denen insgesamt mehr als 30.000 Experten angehören. Trotz ihrer zahlenmäßigen Präsenz übersieht die wissenschaftliche Analyse europäischer Politikprozesse häufig die Expertengruppen, sodass es wenig systematisches Wissen über diese europäische „Schattenverwaltung“ gibt. Formal ist eine Expertengruppe definiert als „ein von der Kommission oder ihren Dienststellen zu Beratungszwecken oder zur Vermittlung von Fachwissen eingesetztes Gremium, dem mindestens sechs Vertreter des öffentlichen und/oder pri- vaten Sektors angehören und das mehr als einmal zusammentritt." "4

Die Expertengruppen beraten die EU-Kommission bei all ihren Gesetzgebungsaufgaben, von der Ausarbeitung von Gesetzgebungsvorschlägen, bis zur Durchführung und Koordinierung von EU Recht. ${ }^{5} \mathrm{Da}$ sie nur beratend tätig sind haben sie keine formale Weisungsbefugnis im europäischen Gesetzgebungsprozess - die EU-Kommission muss den erteilten Rat nicht erhören. Institutionell sind sie bei den Dienststellen der EU-Kommission (Generaldirektionen oder GDs) angesiedelt und sind daher - entsprechend der Organisationslogik der EUKommission - nach Politikressorts gegliedert. Expertengruppen können auf Basis eines Kommissionsbeschlusses (formell) oder durch die Dienststellen in Kooperation mit dem Generalsekretariat (informell) eingesetzt werden, und sie können befristete oder für unbestimmte Zeit berufen werden.

Die Expertengruppen der EU-Kommission sind nach ihrer formalen Definition unbestritten eine technokratische Institution: Zur Partizipation berechtigt in erster Linie Beratungskompetenz oder Fachwissen. Während hier Fachexperten zusammenkommen, bleibt dem „gemeinen Bürger“ der Eintritt verwehrt. Ihre Autorität speist sich also aus ihrem Expertenwissen. Daran ändern auch jüngste Bestrebungen der EUKommission nichts, Expertengruppen nicht als elitär-technokratisches, sondern als partizipatives Element darzustellen (Moodie \& Holst, 2013). Entsprechend urteilte Maurizio Bach über diese Gremien: „Die im Komitee-Regime fest verankerte Definitionsmacht der Experten, seien dies Fachbeamte oder wissenschaftliche Spezialisten, entspricht der modernen Variante der Arkanherrschaft, also technokratischem Regieren." (Bach, 1999: 104)

Anders als Bach argumentiert dieser Beitrag jedoch, dass eine technokratische Institution nicht mit technokratischem Regieren gleichzusetzen ist. Während Expertengruppen von ihrer institutionellen Verfasstheit technokratisch sind, deutet ihre Nutzung durch die EU-Kommission nicht zwangsweise auch auf einen technokratischen Politikprozess hin, bei dem Expertenwissen das Handeln der Akteure bestimmt. Um diese Annahme weiter zu spezifizieren, arbeitet der folgende Absatz drei verschiedene Nutzungsweisen durch die EU-Kommission heraus: eine technokratische, eine strategische, und eine konsensorientierte Nutzung. Die nachfolgenden Ausführungen zeigen, dass sowohl Spezifika des EU-Systems, als auch die Stellung der EU-Kommission im EU-Institutionengefüge die EU-Kommission nicht nur zu einem technokratischen, sondern auch zu einem strategischen Politikstil motivieren. Ent-

4 Siehe http://ec.europa.eu/transparency/regexpert/index.cfm? do=faq.fag\&aide $=2$ \&Lang=DE, zuletzt aufgerufen am 24.4.2012; siehe auch European Commission (2010: 3).

5 Expertengruppen beraten ausschließlich die EU-Kommission. Sie unterschieden sich daher von anderen europäischen Beratungsgremien, wie den formal beratenden „Großen Ausschüssen“ (Europäischer Wirtschafts- und Sozialausschuss oder Ausschuss der Regionen), den Arbeitsgruppen des EU Ministerrats, den Ausschüssen des Europäischen Parlaments oder den 300 Komitologieausschüssen, die EUKommission und Rat bei der Implementation von EU-Recht formal beraten. 
sprechend nutzt sie Expertengruppen nicht nur technokratisch, sondern auch politisch.

\section{Technokratische Nutzung}

Erstens kann die EU-Kommission Expertengruppen durchaus technokratisch, also zur Wissensbeschaffung nutzen. Dies liegt an der technischen Natur der Marktregulierung, die einen auf Expertenwissen basierten Problemlösungsstil des Regierens hervorruft (Radaelli, 1999). Da die EU nur über vergleichsweise geringe eigene Finanzmittel verfügt und somit nur eingeschränkt distributiv tätig werden kann, ist ein Großteil der EU-Politik regulativer Natur. Anders als bei klassischen Nationalstaaten bilden daher nicht finanzielle Leistungen, sondern regulative Maßnahmen (das Festlegen von Verhaltensstandards für die Marktteilnehmer) den Kernbereich des Regierens dar: In der EU ist nicht Geld sondern Expertise die zentrale Steuerungsressource.

Das betrifft auch die EU-Kommission, die als „Motor der Integration“ und „Hüterin der Verträge“ in besonderem Maße auf Expertise angewiesen ist. Wegen ihres bereits erwähnten Personalmangels sind Expertengremien ein Instrument, um der steigenden Nachfrage nach technischer Information und Expertise in einer stetig wachsenden Europäischen Gemeinschaft zu begegnen (Vos, 1999: 19; Mazey \& Richardson, 2006). Hinzu kommt, dass die Umsetzung von EU-Recht meist nicht durch die EU-Kommission selbst, sondern durch nationale oder regionale Verwaltungen erfolgt. Die EU-Kommission ist daher bei der Durchführung von EU-Recht auf die praktische Information der relevanten Sachverständigen aus den Mitgliedstaaten angewiesen, die in Expertengruppen zusammenkommen können.

Die erste Nutzungsweise nimmt also an, dass die Dienststellen der EU-Kommission, einem technokratischen Kalkül folgend, ihre Entscheidungen auf Expertenwissen basieren, das Expertengruppen liefern können. Expertengruppen werden also entsprechend ihrer formalen Definition zu einer sachorientierten Problemlösung genutzt. Hier ist die Nutzung der Expertengruppen durch die EU-Kommission auch Ausdruck technokratischen Regierens.

\section{Strategische Nutzung}

Die EU-Kommission muss jedoch nicht immer als ausführender, technokratischer Akteur agieren, sondern kann auch strategisch handeln. Denn mit ihren weitreichenden Gesetzgebungskompetenzen befindet sie sich inmitten einer Gemengelage aus einer Vielzahl politischer, privater und gesellschaftlicher Akteure. Oft werden ihre Gesetzesvorschläge nicht einfach als neutraler Sachverstand hingenommen, sondern werden politisch angegriffen. Die EU-Kommission wird genötigt, sie ausreichend zu rechtfertigen (ein prominentes Beispiel lieferte die massiv attackierte Dienstleistungsrichtlinie Knill \& Tosun, 2010). Zudem verfolgt die EU-Kommission auch dezidiert eine eigene - ideologisch oder machtpolitisch motivierte - Agenda (Pollack, 1994; Hartlapp et al., 2014, im Erschei- nen). Die Dienststellen der EU-Kommission treten in manche Politikprozesse mit einer bereits vordefinierten inhaltlichen Position ein, die es zu verteidigen gilt. Dieser Lesart zufolge ist die EU-Kommission also nicht nur unabhängige, dem Gemeinwohl verpflichtete Bürokratie, sondern kann durchaus auch politischer Akteur sein, der eigene Interessen verfolgt.

Hier sind Expertengruppen ein geeignetes Mittel, um vordefinierte Positionen argumentativ zu untermauern und legitimieren. Dies geschieht sowohl Kommissions-intern gegenüber konkurrierenden Fachressorts, als auch extern gegenüber den anderen EU-Institutionen. Durch ein unterstützendes Votum anerkannter, „neutraler“ Experten können die vorgeschlagenen Politiklösungen als „rational“ und „unverzerrt“ dargestellt werden (Buholzer, 1998; Pappi \& Henning, 1999). ${ }^{6}$ In solchen Fällen werden politische Entscheidungen nach außen hin als sachorientiert dargestellt. Unter dem Deckmantel der Technokratie ist bei einer solchen Expertengruppennutzung durch die EU-Kommission also tatsächlich ein politisches Kalkül handlungsweisend im Politikprozess.

\section{Konsensorientierte Nutzung}

Ähnlich kann eine dritte, konsensorientierte Nutzungsweise im Kontext politischer Verhandlungen gesehen werden. Expertengruppen können, neben ihrer technokratischen und strategischen Funktion, auch als Foren zur Konsensbildung zwischen relevanten Akteuren des Politikprozesses dienen (Joerges \& Neyer, 1997). Das liegt daran, dass Regieren im EU-Mehrebenensystem nicht nach dem Mehrheitsprinzip, sondern nach dem Prinzip der „checks and balances“ organisiert ist. Da die politischen Akteure nicht auf stabile Mehrheiten vertrauen können, beruht europäische Entscheidungsfindung in erster Linie auf einer kontinuierlichen Konsensfindung (Jachtenfuchs, 2008). In diesem Kontext fällt der EUKommission oft die Rolle des „honest broker“ (etwa: Mediator oder ehrlicher Vermittler Cini, 1996: 28 ff.) unter widerstreitenden Interessen zu. Zum einen kann die EU-Kommission als supranationale Institution zwischen den Mitgliedstaaten, und zum anderen als „neutrale“ Bürokratie zwischen den beiden politischen EU-Gesetzgebungsorganen Rat und Parlament vermitteln. Die EU-Kommission ist dabei selbst an einer effektiven Konsensfindung interessiert, da der Erfolg der federführenden Dienststellen oft daran gemessen wird, wie reibungslos ihre Gesetzesinitiativen den politischen Prozess durchlaufen und erfolgreich in der Implementation sind (Laffan, 2002).

Demnach sind Expertengruppen in Antizipation des nachfolgenden Politikprozesses nützliche Foren, um Konsens in einem Politikfeld zu schaffen und relevante Veto-Spieler

6 Im Vergleich zur technokratischen Nutzung ist die EU-Kommission bei der Vorbereitung von Gesetzesvorlagen nicht offen für externe Expertise, sondern filtert jene Information selektiv heraus, die ihren Zwecken dienlich ist. Entsprechend sollten strategisch genutzte Expertengruppen weniger Einfluss auf die Gesetzesvorschläge haben, da die von der EU-Kommission präferierte Politiklösung schon steht und es keinen tatsächlichen Bedarf für zusätzlichen Input gibt (so auch Radaelli, 1999). 
„weichzukochen“ (Larsson, 2003). Beispielsweise sitzen in manchen Expertengruppen nationale Ministerialbürokraten, die in ähnlicher Besetzung später in den Arbeitsgruppen des Ministerrats bei der Verabschiedung der Gesetze mitwirken, sodass hier Meinungen vorsondiert werden können (Egeberg et al., 2003). Bei dieser Nutzungsweise ruft der konsensorientierte Entscheidungsstil des EU-Systems bei der EU-Kommission eine Nutzung von Expertengruppen als Verhandlungsarenen hervor (Jachtenfuchs, 2008). Ähnlich wie bei der strategischen, deutet auch die konsensorientierte Nutzung von Expertengruppen durch die EU-Kommission eher auf einen politischen als auf einen technokratischen Politikprozess hin.

Die verschiedenen Nutzungsweisen zeigen also, dass die Einbindung von Expertengruppen in die Politikgestaltung ungeachtet ihres technokratischen Mandats nicht zwangsläufig auf einen technokratisch motivierten Politikprozess hinweist.

\section{Das Expertengruppensystem im quantitativen Überblick}

Im Folgenden wird ein quantitativer Überblick über die Konstitution des Expertengruppensystems der EU-Kommission gegeben. Die gemeinhin angenommene These, dass die Expertengruppen zur Wissensakquise genutzt werden und daher auf technokratisches Regieren hinweisen, findet hier Unterstützung, wenn folgende Hypothesen bestätigt werden: Erstens, über Zeit und mit einer zunehmenden Regulierungsdichte steigt die Anzahl an Expertengruppen. Zweitens, es gibt mehr Expertengruppen in regulativen als in distributiven Politikfeldern.

\section{Entwicklung über Zeit}

Eine technokratische Gremiennutzung sollte sich in einem Anstieg von Expertengruppen über Zeit widerspiegeln. Denn mit wachsenden supranationalen Kompetenzen über Zeit kommt es zu einer steigenden Arbeitsbelastung der EU-Kommission und einer entsprechenden Nachfrage nach externem Fachwissen. Die Daten für die quantitative Analyse wurden größtenteils aus dem „Expertengruppenregister“ der EUKommission ausgelesen. ${ }^{7}$ Seit 2005 stellt die EU-Kommission darin eine Vielzahl von Informationen zu ihren aktuell konsultierten Expertengruppen bereit. Expertengruppen gibt es bei der EU-Kommission jedoch schon viel länger als dieses Register. So äußerte ein Interviewpartner aus der GD Forschung der EU-Kommission: "Expertengruppen gab es vom ersten Tag an; ich glaube Gott hat Eva und Adam erschaffen, und dann eine Expertengruppe. “ (COM136:166, eigene Übersetzung). ${ }^{8}$ In der Tat weisen die Gründungsverträge der EU darauf hin, dass die EU-Kommission seit Anbeginn ihrer Tätigkeit mit Expertengruppen arbeitet. Abbildung 1 zeigt die zahlenmäßige Entwicklung der Expertengruppen über Zeit.
Abbildung 1: Anzahl der Expertengruppen im Zeitverlauf (1985-2010)

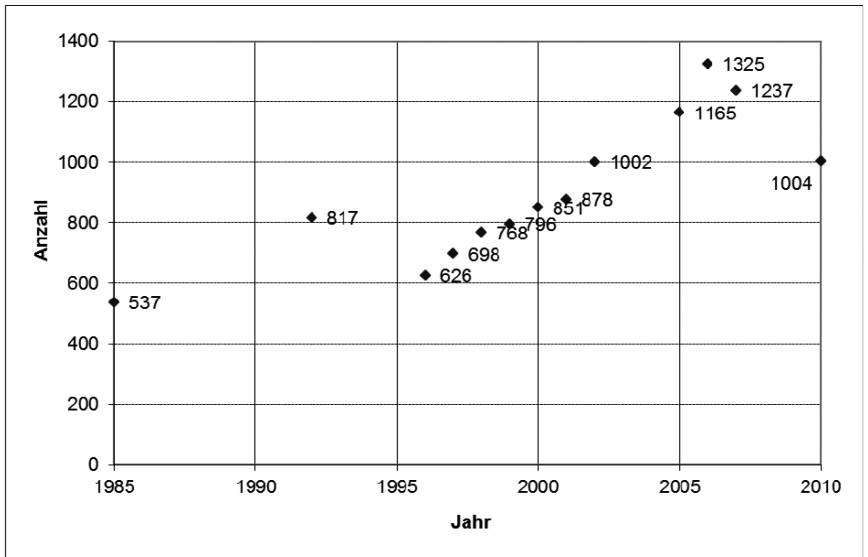

Daten: Jahre 1985, 1992, 1996-1999 und 2000-2002: Brochhagen (2010); Jahr 2000: Larsson und Murk (2003); Jahr 2005: Broscheid und Coen (2007); Jahr 2007: Gornitzka und Sverdrup (2008); Jahr 2010: Expertengruppenregister, Daten erhoben im Februar 2010

Ausgenommen eines Ausreißers im Jahr 1992 zeigt die Abbildung einen kontinuierlichen Zuwachs des Gremienwesens, der im Jahr 2006 gipfelt, und anschließend wieder fällt. Beim Vergleich der einzelnen Datenpunkte muss jedoch auf eine beschränkte Datenreliabilität hingewiesen werden, da sich die formale Definition einer „Expertengruppe“ über Zeit veränderte. So beinhaltete eine Reform der internen Rahmenregelung im Jahr 2005 eine Neudefinition von Expertengruppen, die im Jahr 2011 erneut überarbeitet wurde. Da das offizielle Expertengruppenregister zudem erst 2005 eingeführt wurde, beruhen Daten aus früheren Jahren auf alternativen Quellen. Beispielsweise dienten die jährlichen Haushalsberichte der EUKommission von 1998 bis 2002 als Basis für die interne Expertengruppenverwaltung (für eine Diskussion der historischen Entwicklung des internen Regelungsrahmens siehe Metz, 2012).

Entsprechend der konzeptionellen Annahme deutet der Gremienzuwachs über Zeit (bis 2006) auf eine technokratische Expertengruppennutzung hin. Weiterführende Studien konnten diesen Mechanismus jedoch nicht belegen: Besonders viele Expertengruppen gibt es heute nicht in Politikbereichen mit supranationalen Kompetenzen - also dort, wo die Regelungsdichte sehr hoch ist - sondern mit geteilten Kompetenzen, wo viel Koordinierungsbedarf besteht (Gornitzka \& Sverdrup, 2008).

Eine alternative Erklärung für den Zuwachs über Zeit kann paradoxerweise das Konzept der politischen Expertengruppennutzung liefern. Denn die EU-Integration über Zeit ging auch mit einer zunehmenden Politisierung der EU einher (Hooghe \& Marks, 2009, siehe auch Einleitung). So wurde in den 1990er Jahren in Folge einer verstärkten Integration durch

7 Siehe http://ec.europa.eu/transparency/regexpert/index.cfm? Lang=DE, zuletzt aufgerufen am 24.4.2013.

8 Interviews werden zitiert als COMO1, COMO2, usw. (für EU-Kommissionsbeamte) oder EXPO1, EXPO2, usw. (für Expertengruppenmitglieder) und beinhalten den genauen Paragraphen des jeweiligen Interviewtranskripts (siehe auch Kapitel vier zur qualitativen Datenerhebung). 
den Maastricht Vertrag der zuvor herrschende „permissive Konsensus“ durch eine zunehmende Infragestellung und Kritik an der EU abgelöst, wovon auch die EU-Kommission nicht gefeit blieb. Wenn man von einer strategischen und konsensorientierten Nutzung von Expertengruppen ausgeht, kann der beobachtende Zuwachs des Expertenwesens also auch durch die Politisierung der EU erklärt werden.

Der zu beobachtende Abfall nach 2006 ist als Folge einer internen Evaluation zu deuten, die 2007 vom Generalsekretariat der EU-Kommission lanciert wurde. Die interne Evaluation sollte die Reliabilität der Daten steigern, Bürokratie abbauen und $\mathrm{zu}$ mehr Transparenz führen. Unter anderem sollte das Löschen aller „Karteileichen“ dazu beitragen, „die Anzahl von Expertengruppen maßgeblich auf ungefähr 1000 zu reduzieren " (European Commission / Secretariat-General, 2009: 6, eigene Übersetzung). Der starke Abfall von 23\% im Zeitraum zwischen 2007 und 2010, der parallel zum Evaluierungsprozess stattfand, ist also größtenteils interner Reformen geschuldet und spiegelt nicht einen abnehmenden Beratungsbedarf der EU-Kommission wider. Er kann daher weniger im Lichte der konzeptionellen Überlegungen zur technokratischen und politischen Nutzungsweise gedeutet werden.

\section{Verteilung über Politikressorts}

Eine technokratische Nutzung der Expertengruppen durch die EU-Kommission sollte des Weiteren in zahlenmäßigen Unterschieden zwischen Politikfeldern zu beobachten sein. Nutzt die EU-Kommission ihre Expertengruppen in erster Linie technokratisch, also auf Basis deren Expertise, sollte es eine verstärkte Expertengruppennutzung in den Politikressorts geben, die mehr Expertise benötigen. Wie im konzeptionellen Teil ausgeführt, kann angenommen werden, dass regulative Politiken im Vergleich zu distributiven in besonderem Maße auf Expertenwissen angewiesen ist. Für die folgende Analyse wurden die Dienststellen der EU-Kommission daher entsprechend ihres Politikressorts binär als regulativ oder distributiv klassifiziert ${ }^{9}$

Abbildung 2: Verteilung der Expertengruppen über EU-Kommissionsdienststellen

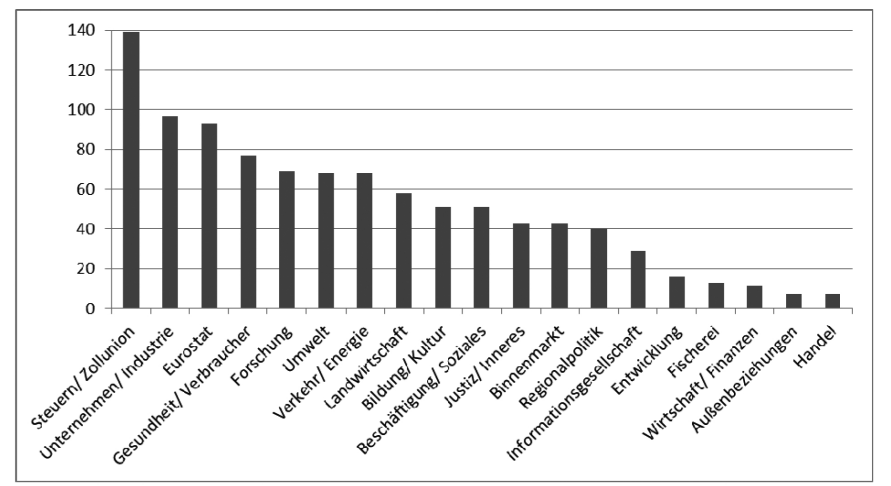

Daten: Expertengruppenregister, erhoben im Februar $2010(N=1014)$ Anmerkung: Nur Dienststellen mit mindestens fünf Expertengruppen abgebildet
Die Verteilung in Abbildung 2 lässt vermuten, dass es keinen Zusammenhang zwischen der regulativen Natur eines Politikfeldes und der Anzahl an Expertengruppen gibt: Die ressourcenstärksten distributiven Ressorts in der EU-Kommission Landwirtschaft, Regionalpolitik, Forschung und Beschäftigung/ Soziales ${ }^{10}$ - sammeln sich alle im mittleren Bereich der Grafik und nicht im unteren Bereich mit wenigen Expertengruppen. Auch statistisch gibt es keinen signifikanten Zusammenhang. Zwar ist der Zusammenhang zwischen der distributiven Natur eines Politikfelds und der Anzahl von Expertengruppen negativ $(\mathrm{R}=-0.0624)$ - das heißt distributive Politikressorts haben in der Tat weniger Expertengruppen -, doch ist er nicht signifikant (zu einem ähnlichen Ergebnis auf Basis von Daten aus früheren Jahren kommen auch Broscheid \& Coen, 2007; Gornitzka \& Sverdrup, 2008). ${ }^{11}$

Die quantitativen Daten lassen also keine Häufung von Expertengruppen in regulativen Politikressorts erkennen. Ebenso wie die Entwicklung über Zeit findet die These einer vorwiegend technokratischen Nutzung durch die EU-Kommission empirisch keine handfeste Unterstützung. Besonders die aufgeführten Bedenken gegenüber den quantitativen Daten und deren ambivalente Interpretation erfordern einen tieferen Einblick in die Materie, welcher nachfolgend genommen wird.

\section{Die Rolle der Expertengruppen im Politikprozess}

Die qualitative Untersuchung der Nutzung der Expertengruppen bei der Gesetzesvorbereitung basiert auf empirischen Daten eines mehrjährigen kollaborativen Forschungsprojekts zur „Positionsbildung in der EU-Kommission“ am Wissenschaftszentrum Berlin für Sozialforschung (WZB). Hier wurden 48 Politikformulierungsprozesse in der EU-Kommission im Zeitraum von 1999 bis 2008 in drei Querschnittspolitikfeldern untersucht - der Schnittstelle zwischen Sozial- und Binnenmarktpolitik (16 Gesetzesvorschläge), Forschungsund Innovationspolitik (14) und Verbraucherpolitik (18). Zu jeder dieser Gesetzesinitiativen wurde in einer detaillierten Fallstudie der Gesetzformulierungsprozess in der EU-Kommission aufgearbeitet. Als Datenbasis dienten vorwiegend 149 halb-standardisierte Interviews mit Kommissionsbeamten (138) und Expertengruppenmitgliedern (11), sowie Primärdokumente (etwa Gesetzestexte, vorläufige Gesetzesentwürfe

9 Die Klassifizierung erfolgt in Anlehnung an Broscheid und Coen (2007). Distributiv: Beschäftigung/ Soziales, Bildung/ Kultur, Entwicklung, Fischerei, Forschung, Handel, Landwirtschaft, Regionalpolitik, Streuern/ Zollunion. Wie die Autoren anmerken, ist die Einordnung nicht unstrittig, da viele Dienststellen sowohl regulativ als auch distributiv tätig sind (siehe auch Fußnoten 10 und 11)

10 Die GD Beschäftigung/ Soziales ist wegen ihrer Verwaltung des Europäischen Sozialfonds als „distributiv“ klassifiziert (Broscheid \& Coen, 2007: 354).

11 Bei der Klassifizierung verwundert insbesondere Broscheid und Coens (2007) Einordnung der GD Steuern/ Zollunion als distributiv (siehe Anhang). Da diese Generaldirektion am oberen Ende mit 139 Expertengruppen angesiedelt ist und daher den statistischen Zusammenhang maßgeblich beeinflussen kann, wurde hier auch der Zusammenhang für eine Kodierung der GD Steuern/Zollunion als regulativ getestet. Der negative Zusammenhang ist dann zwar stärker ( $R=$ -0.3363), aber immer noch insignifikant. 
und andere interne Dokumente, Gutachten und Analysen, Positionspapiere, Zeitungsartikel).

In den 48 Fallstudien wurde unter anderem die Nutzung von Expertengruppen durch die zuständigen Dienststellen bei der Gesetzesvorbereitung systematisch analysiert. Dieses Kapitel gibt erst einen zusammenfassenden Überblick über die Art der Expertengruppennutzung und illustriert diese anschließend anhand beispielhafter Fallstudien.

In drei Viertel der untersuchten Fälle (35 von 48) involvierte die EU-Kommission Expertengruppen in die Gesetzesvorbereitung. Oftmals konsultierten die Dienststellen bei der Vorbereitung eines Gesetzestextes sogar mehrere Gremien parallel - und das, obwohl die Anhörung von Expertengruppen für die EU-Kommission nur in seltenen Fällen verpflichtend ist. ${ }^{12}$

Tabelle 1: Art der Expertengruppennutzung in der EU-Kommission (Anteil aller Fälle bei denen eine Expertengruppe effektiv genutzt wurde, $\mathrm{N}=35$ )

\begin{tabular}{|c|c|c|}
\hline \multicolumn{3}{|c|}{ Art der Expertengruppennutzung } \\
\hline Technokratisch & Strategisch & Konsensorientiert \\
\hline $66 \%$ & $51 \%$ & $26 \%$ \\
\hline
\end{tabular}

Tabelle 1 gibt Auskunft über die Art der Expertengruppennutzung in den 35 Fällen in denen konsultiert wurde. In zwei Drittel der Fälle wurden Expertengruppen technokratisch genutzt, gefolgt von einer strategischen Nutzung in etwas mehr als der Hälfte aller Fälle und einer Konsensbildung in 26 Prozent. Das bedeutet, dass die EU-Kommission Expertengruppen meistens entsprechend des technokratischen Mandats, zur Wissensbeschaffung, nutzte. Doch in fast allen dieser Fälle fand parallel auch eine strategische Nutzung statt. Selten waren die untersuchten Politikprozesse ausschließlich technokratisch. Vielmehr agierten die Dienststellen bei der Gesetzesvorbereitung sowohl technokratisch als auch politisch.

Erklären lässt sich das zum Großteil durch die Natur des Regelungsinhalts - maßgeblich der technischen Komplexität und der Strittigkeit - die das Handlungskalkül der Generaldirektionen beeinflusste. Und da Gesetzestexte oft sowohl technische Details als auch kontroverse politische Punkte enthalten, kam es innerhalb eines Prozesses zur unterschiedlichen Nutzung einer oder mehrerer Expertengruppen. Während die Arten der Expertengruppennutzung analytisch Idealtypen darstellen, können sie sich in der Realität also überschneiden. Das zeigen auch die Prozentzahlen, die aufgrund verschiedener Nutzungsweisen bei nur einem Gesetzestext zusammengenommen mehr als 100 Prozent ergeben.

So konsultierte die GD Binnenmarkt bei der Vorbereitung zweier kontroverser und zeitgleich technisch komplexer Direktiven zum Vergaberecht (KOM[2000]275 und KOM[2000]276) zwei formal eingesetzte Expertengruppen aus dem Bereich des öffentlichen Auftragswesens (COM16:176, 57). Der Beratende Ausschuss für das öffentliche Auftragswesen (71/306/EWG) bestand aus Vertretern der
Mitgliedstaaten, der Beratende Ausschuss für die Öffnung des öffentlichen Auftragswesens (87/305/EWG) war ein gemischtes Gremium aus Interessenverbänden und wissenschaftlichen Sachverständigen. In beiden Gremien saßen vorwiegend Mitglieder mit langjähriger Erfahrung und Expertise im nationalen Vergabewesen. Wie das folgende Zitat verdeutlicht, nutzte die GD Binnenmarkt ihre Expertengruppen sowohl für die Entwicklung technischer Indikatoren als auch zur strategischen Unterfütterung ihrer Position gegenüber Mitgliedstaaten:

„Wir haben mit den Expertengruppen darüber diskutiert, wie wir Indikatoren entwickeln können, um Statistiken darüber zu bekommen, wie wir die Sache angehen. [...] Da gab es natürlich auch Überschneidungen, die Gruppe auch dazu zu nutzen, um unserer Position im Rat zu helfen. Die beiden Dinge waren also nicht isoliert; selbstverständlich konnte man den Text in den Expertengruppen nicht isolieren." (COM22:136, eigene Übersetzung)

Diese Ausführung ist beispielhaft dafür, wie eine Generaldirektion eine Expertengruppe sowohl technokratisch als auch politisch nutzt. Das war dadurch bedingt, dass die Gesetzestexte sowohl technische Details enthielten, deren Ausarbeitung externen Sachverstand benötigte, als auch kontroverse Punkte, deren Verteidigung externer Unterstützung bedurfte.

Eine technokratische Nutzung war über alle Politikfelder gleichmäßig verteilt. Sie trat insbesondere dann auf, wenn die Dienststellen für die Ausarbeitung technischer Einzelheiten eines Gesetzes nicht genügend internes Fachwissen hatten. Ein Beispiel hierfür ist die von der GD Binnenmarkt vorgeschlagene Verordnung zur Verkaufsförderung (KOM[2001]546) aus dem Verbraucherschutzbereich, die die grenzüberschreitende Verkaufsförderung von Produzenten an Konsumenten regulieren sollte. In diesem Fall fand die GD Binnenmarkt eine heterogene Landschaft unterschiedlicher Verbraucherschutzniveaus in den Mitgliedstaaten vor. Um die nötige Information über die national bestehenden Beschränkungen der Verkaufsförderung zu erhalten, berief sie eine Expertengruppe bestehend aus Rechtsexperten aus den Mitgliedstaaten ein (COM79; COM133). Das Gremium lieferte in seinem Abschlussbericht eine umfassende Übersicht der nationalen Vorschriften in den damals 15 EU-Mitgliedstaaten. Die GD Binnenmarkt nutzte das Ergebnis als Basis für ihre Vorschläge, welche nationalen Regelungen aufgehoben, welche dem Prinzip gegenseitiger Anerkennung und welche unter der neuen Verordnung harmonisiert werden sollten. Der Fall zeigt, wie die EU-Kommission eine Expertengruppe einberief, um erforderliches Fachwissen zu erhalten. Aufgrund des Informationsbedarfs der zuständigen Dienststelle dominierte das Experten-

12 Zudem können die Dienststellen neben Expertengruppen auch auf eine Vielzahl anderer Beratungskanäle zurückgreifen, etwa Auftragsstudien durch Beratungsfirmen, öffentliche Konsultationen, die Neueinstellung abgeordneter nationaler Sachverständige, Workshops und Konferenzen oder der bilaterale Austausch mit individuellen Beratern wie den persönlichen Beratern der Kommissare. 
wissen der Gremienmitglieder den Beratungsprozess und weist auf einen technokratischen Politikprozess hin.

Eine strategische Expertengruppennutzung tauchte am häufigsten im Bereich der Forschungs- und Innovationspolitik auf, einem vorwiegend distributiven Politikfeld. Oft war hier die Programmkonzipierung in der EU-Kommission von politischen Verteilungskämpfen zwischen den einzelnen Ressorts geprägt. Zudem benötigten die federführenden Generaldirektionen in Antizipation anstehender kontroverser Auseinandersetzungen mit Rat und Parlament die externe Unterstützung von Expertengremien für ihre Budgetforderungen.

Ein Beispiel einer Expertengruppe, die strategisch genutzt wurde, ist der Europäische Forschungsbeirat der GD Forschung (European Research Advisory Board, EURAB, 2001/531/EC, Euratom), ein formales Gremium mit namhaften Persönlichkeiten aus Wissenschaft und Industrie. Formal damit beauftragt, die EU-Kommission in Forschungsfragen inhaltlich zu beraten, wurde der Forschungsbeirat auch dazu genutzt, „großen politischen Projekten eine kritische Masse zu liefern“ (COM136:134, eigene Übersetzung). Das wurde nötig als die GD Forschung für das Siebte Forschungsrahmenprogramm (RP7, 2007-2013, KOM[2005]119-1) eine Verdopplung des EU Forschungsbudgets forderte. Bereits während der Kommissions-internen Vorbereitungen zeichneten sich die mitgliedstaatlichen Positionen entsprechend einer „juste retour“ Logik ab: Großbritannien etwa war strikt gegen eine Ausweitung des gesamten EU-Haushalts, Frankreich fürchtete Einbußen für den Landwirtschaftsetat (COM10:10). In dieser politischen Gemengelage war die GD Forschung um jede Art argumentativer Unterstützung für ihre weitreichenden Budgetforderungen dankbar. EURAB, selber auch an einem höheren Forschungsetat interessiert, war ein willkommenes Instrument, um die politische Forderung der EU-Kommission wissenschaftlich zu unterfüttern. Zeitgleich mit der Vorlage des Gesetzesentwurfs zum RP7 im April 2005 verfasste EURAB einen Bericht, wo er die Dringlichkeit einer Verdopplung des Forschungsetats für die europäische Wissenschaft und Industrie Nachdruck darlegte: Für die Forschung in Europa sei dies ein „Minimalziel“, um den aktuellen internationalen Herausforderungen zu begegnen (EURAB 05.015, April 2005; auch EXP07). Mit seinem unterstützenden Expertenvotum diente das hochrangige Gremium ganz klar strategischen Zwecken. Der Fall ist beispielhaft für einen politischen Verhandlungsprozess, bei dem die Meinung von Experten als politische Munition genutzt wird.

Eine konsensorientierte Expertengruppennutzung tauchte vor allem bei Gesetzesvorbereitungen im Schnittstellenbereich Sozialpolitik/ Binnenmarktpolitik auf wo historisch gewachsene nationale Unterschiede viel Konfliktpotenzial bargen. Zum Beispiel sollte der Richtlinienvorschlag der GD Binnenmarkt zur Beaufsichtigung von Finanzkonglomeraten (KOM[2001]213) Schlupflöcher im heterogenen sektoralen Recht der Mitgliedstaaten schließen. ${ }^{13}$ Ziel des Vorschlags war es, den Markt zu stabilisieren, die Aufsicht zu regulieren und den Schutz von Anlegern, Sparern und Inhabern von
Lebensversicherungen zu verbessern. Um für dieses hochtechnische Gesetz das nötige Fachwissen zu erwerben, stellte die Generaldirektion einen abgeordneten nationalen Sachverständigen neu ein (aus dem Mitgliedstaat, dessen nationaler Rechtsrahmen als Vorlage für den Kommissionsvorschlag diente).

Andererseits war der Regulierungsvorstoß der GD Binnenmarkt politisch hochsensibel. Erstens herrschten heterogene nationale Ausgangsbedingungen, und zweitens galten die zu regulierenden Finanzkonglomerate als elementar für die wirtschaftliche Stabilität der Mitgliedstaaten. Da die Generaldirektion ihre Initiative zudem auf dem Rechtsrahmen eines einzelnen Mitgliedstaates basierte, führte dies zu Kontroversen zwischen den Mitgliedstaaten und innerhalb des Finanzsektors. Daher errichtete die Generaldirektion eine heterogen zusammengesetzte Expertengruppe mit Sachverständigen aus verschiedenen Finanzbereichen und Mitgliedstaaten, um auf einen Konsens für ihren präferierten Regulierungsansatz hinzuarbeiten:

„Ich glaube, dass es im Zuge dieser Richtlinie und der Diskussionen, die um die Gesetzesinitiative stattfanden, das erste Mal war, dass diese verschiedenen Aufsichtsbehörden zusammen im gleichen Raum saßen. Daher war es eine sehr schwierige Diskussion. Und es war wichtig, das im Kopf zu behalten, um während des Prozesses so etwas wie einen gemeinsamen Standpunkt zu entwickeln." (COM76:36, eigene Übersetzung).

Hier wird deutlich, wie die EU-Kommission bewusst eine heterogen zusammengesetzte Expertengruppe einsetzte, um einen Konsens um „einen gemeinsamen Standpunkt zu entwickeln“. Wie im vorherigen Beispiel auch, zeugt das - trotz Expertengruppeneinbindung - weniger von technokratisch als von politisch motivierten Akteuren im Politikprozess.

\section{Expertengruppen als Inbegriff technokratischen Regierens?}

Die Erkenntnisse der empirischen Analysen weisen die These zurück, dass die EU-Kommission ihre Expertengruppen vorwiegend technokratisch nutzt und zeigen auf, dass es auch eine politische Nutzung dieser Gremien geben muss. Während Expertengruppen zwar technokratische Institutionen sind, werden sie in politisch motivierten Politikprozessen auch strategisch genutzt. Insbesondere die Fallstudien deuten darauf hin, dass Charakteristika des zu regulierenden Sachverhalts bei der EU-Kommission unterschiedliche Handlungskalküle hervorrufen. Je nach technischer Komplexität, politischer Strittigkeit oder finanzieller Implikationen werden Expertengruppen zur Problemlösung, zur Konsensfindung oder zur Legitimierung genutzt. Ebenso liefern Experten nicht nur neutrale Expertise, sondern nutzen ihre technokratische Position auch, um Lobbying zu betreiben.

13 Die Häufung der gewählten Beispiele unter Federführung der GD Binnenmarkt ist rein zufälliger Natur. Empirisch nutzte diese Generaldirektion Expertengruppen nicht häufiger als andere Dienststellen. 
Hierbei ist politisch als Gegensatz zu technokratisch jedoch nicht mit demokratisch oder partizipatorisch gleichzusetzen. Denn eine politische Nutzung von Expertengruppen darf nicht darüber hinwegtäuschen, dass auch hier die zentrale Legitimationsbasis bei der Nutzung von Expertengruppen deren Expertise ist - nur wird sie eben strategisch verwendet. Expertengruppen sind von ihrem Wesen und Auftrag her Sachverständigenbeiräte und nicht partizipatorische Elemente zur Bürgerbeteiligung.

Damit sind sie natürlich auch ein Stück weit intransparent und elitär, was der EU-Kommission zunehmend zum Vorwurf gemacht wird (Moodie \& Holst, 2013). Das wird besonders im Lichte der von vielen privaten, gesellschaftlichen und politischen Akteuren wahrgenommenen steigenden Intransparenz des Europäischen Gesetzgebungsprozesses als problematisch gesehen (Mahony, 2013). So hat das Europäische Parlament jüngst einen Teil des für Expertengruppen vorgesehenen EUBudgets so lange eingefroren, bis die EU-Kommission bei der Transparenz und Offenheit ihrer internen Rahmenregelung für Expertengruppen nachlegte (European Parliament / Committee on Budgets, 2011).

Wenn über das Paradigma der Brüsseler Technokratie referiert wird, muss natürlich auch gefragt werden, inwiefern das Gremienwesen der EU-Kommission spezifisch für die EU ist, oder ob es auf nationaler Ebene ähnlich ausgeprägte Beratungsstrukturen gibt. In der Tat zeigen mehrere Studien, dass nationale Regierung in einem ähnlichem Ausmaß und auch in ähnlicher Weise Expertengremien für verschiedene Zwecke nutzen (etwa für Deutschland Siefken, 2007; Weingart \& Lentsch, 2008: 119; für Frankreich Murswieck, 2006: 590; für die USA Zegart, 2004; Brown, 2008: 547; für Kanada Howlett \& Maragna, 2006). Ein (stabiles) Netz an Beratungsgremien ist für das Regieren auf nationaler wie auf supranationaler Ebene ein wesentliches und zahlenmäßig bedeutsames Element der Politikberatung.

Auf EU-Ebene stellt sich die Sachlage jedoch trotzdem etwas anders dar. Denn wohingegen Expertengremien auf der nationalen Ebene demokratisch gewählte Regierungen beraten, assistieren die hier untersuchten Expertengruppen die EUKommission, die selber eine technokratische Institution ist. Bei deren Zusammenspiel kann man also in der Tat von einer „expertokratischen Funktionselite“ (Bach, 1999: 54-5) sprechen. Doch trotz ihres technokratischen Erscheinungsbilds orientiert sich diese Elite nicht ausschließlich an sachorientierten Argumenten sondern reagiert auf den jeweiligen Politikkontext. Trotz ihres elitären Charakters handelt die Brüsseler Technokratie nicht losgelöst von den Kontextbedingungen des Politikprozesses und nimmt diese bereits bei der internen Gesetzesvorbereitung auf.

\section{Konklusion}

In der aktuellen Eurokrise ist der Vorwurf eines von Experten dominierten, elitären und intransparenten europäische Politikprozesses nochmal verschärft worden (Walter, 2012). Wie dieser Beitrag zeigt, verbergen sich hinter einer technokrati- schen Erscheinung häufig politische Prozesse. Die EU-Kommission handelt in Interaktion mit Expertengruppen nicht ausschließlich sachorientiert, sondern durchaus auch als politischer Akteur, indem sie Experten als Instrument zur Legitimation ihrer Vorhaben und zur Konsensbeschaffung nutzt. Expertengruppen dienen daher nicht nur der Bereitstellung von Expertise, sondern tragen in vielfältiger Weise zum Politikprozess bei. Meist sind Unterschiede in der Nutzungsweise durch Charakteristika der Regulierungsmaßnahme bedingt. Eine politische Nutzung ist auch im Kontext des folgenden inter-institutionellen Prozesses zu sehen. Brüsseler Technokratie und politische Arena stehen also nicht als zwei Parallelwelten voneinander abgekoppelt $\mathrm{da}$, sondern sind miteinander verwoben.

Zudem muss man zwischen der technokratischen Beschaffenheit der Institutionen und einem technokratischen Handlungskalkül im Politikprozess unterscheiden. Denn während das Expertenwesen der EU-Kommission durchaus eine technokratische Institution darstellt, darf ihre Einbindung in die Politikgestaltung nicht über einen möglicherweise hochpolitischen Prozess hinwegtäuschen. Eine Schärfung unseres Verständnisses für den europäischen Politikprozess jenseits von Stereotypen erfordert daher einen Blick hinter die Kulissen formaler Institutionen und die systematische Analyse informeller Strukturen, wie den Expertengremien.

\section{Literatur}

Bach, M. (1999). Die Bürokratisierung Europas: Verwaltungseliten, Experten und politische Legitimation in Europa. Frankfurt/ New York: Campus.

Bittner, J. (2012). Gute Zeiten für Lobbyisten. Die ZEIT. 6.12.2012 Nr. 50 .

Brochhagen, L. (2010). Seeking Advice in a Multi-Level Governance System - a Sectoral Analysis of the Composition of Expert Groups to the European Commission. Magisterarbeit an der Philosophischen Fakultät der Universität zu Köln.

Broscheid, A. \& Coen, D. (2007). Lobbying activity and fora creation in the EU: empirically exploring the nature of the policy good, Journal of European Public Policy, 14(3), 346 - 365

Brown, M. B. (2008). Fairly Balanced: The Politics of Representation on Government Advisory Committees, Political Research Quarterly, 61(4), 547-560.

Buholzer, R. (1998). Legislatives Lobbying in der Europäischen Union: Ein Konzept für Interessengruppen. Bern: Verlag Paul Haupt.

Cini, M. (1996). The European Commission: Leadership, organization and culture in the EU administration. Manchester: Manchester University Press.

Egeberg, M., Schäfer, G. F. \& Trondal, J. (2003). The many faces of EU committee governance, West European Politics, 26(3), 19-40.

European Commission (2010). Communication from the President to the Commission: Framework for the Commission expert groups: framework for Commission expert groups: horizontal rules and public register. C(2010) 7649 final. Brussels, 10.11.2010.

European Commission / Secretariat-General (2009). Note to Directors Generals and Heads of Services - Subject: Evaluation report on the horizontal rules within the framework for Commission's expert groups. SEC(2009)486, SG.E.1/AM/ptp D(2009)2644. Brussels, 2.4.2009.

European Parliament / Committee on Budgets (2011). Amendment 534 on Francesca Balzani's report on the 2012 EU Budget. VERT/8614. Strasbourg, 12.10.2011. 
Follesdal, A. \& Hix, S. (2006). Why There is a Democratic Deficit in the EU: A Response to Majone and Moravcsik, JCMS: Journal of Common Market Studies, 44(3), 533-562.

Gornitzka, Å. \& Sverdrup, U. (2008). Who consults? The configuration of expert groups in the European union, West European Politics, 31(4), $725-750$.

Haas, P. M. (1992). Introduction: Epistemic Communities and International Policy Coordination, International Organization, 46(1), 1-35.

Hartlapp, M., Metz, J. \& Rauh, C. (2014, im Erscheinen). Which policy for Europe? Power and conflict inside the European Commission. Oxford: Oxford University Press.

Hartleb, F. (2011). A thorn in the side of European elites: The new Euroscepticism. Brussels: Centre for European Studies.

Hooghe, L. \& Marks, G. (2009). A Postfunctionalist theory of European integration: From permissive consensus to constraining dissensus., British Journal of Political Science, 39(1), 1-23.

Howlett, M. \& Maragna, A. (2006). How Big Is a Policy Network? An Assessment Utilizing Data From Canadian Royal Commissions 19702000, Review of Policy Research, 23(2), 433-452.

Jachtenfuchs, M. (2008). Institutionelle Struktur und Governance in der EU, In: Schuppert, G. F.Zürn, M. (Hrsg.), Governance in einer sich wandelnden Welt. Wiesbaden: VS Verlag, 383-400.

Joerges, C. \& Neyer, J. (1997). From Integrovernmental Bargaining to Deliberative Political Processes: The Constitutionalisation of Comitology, European Law Journal, 3(3), 273-299.

Kaube, J. (2012). Technokratie und Managerherrschaft. Zur Theoriegeschichte eines schlimmen Verdachts, MERKUR, 09/10(September 2012), 857-865.

Knill, C. \& Tosun, J. (2010). Politikgestaltung in der Europäischen Union. Die Entstehung und Umsetzung der Dienstleistungsrichtlinie. BadenBaden: Nomos.

Laffan, B. (2002). The European Commission: Promoting EU Governance, In: Grote, J. R.Gbikpi, B. (Hrsg.), Participatory Governance. Political and Societal Implications. Opladen: Leske + Budrich, 121-139.

Larsson, T. (2003). Precooking - The World of Expert Groups. Stockholm: ESO, Fritzes Offentliga Publikationer.

Mahony, H. (2013). Complex EU law-making dubbed 'infernal, undemocratic'. EUobserver. Brussels, 04.03.13.

March, J. G. \& Olsen, J. P. (1989). Rediscovering Institutions: The Organizational Basis of Politics. New York: The Free Press, Maxmillan.

Mazey, S. \& Richardson, J. (2006). The Commission and the lobby, In: Spence, D. (Hrsg.), The European Commission. London: John Harper, 279-92.

Metz, J. (2012). The European Commission's expert groups and their use in policy making. unpublished PhD thesis. Berlin, FU Berlin.

Moodie, J. \& Holst, C. (2013). For the Sake of Democracy? The European Commission's Justifications for Democratizing Expertise. EPISTO kickoff conference. Oslo, 4-5 April 2013.

Murswieck, A. (2006). Politikberatung in Frankreich, In: Falk, S., Thunert, M., Rehfeld, D., et al. (Hrsg.), Handbuch Politikberatung. Wiesbaden: VS Verlag für Sozialwissenschaften, 590-89.
Pappi, F. U. \& Henning, C. H. C. A. (1999). The organization of influence on the EC's common agricultural policy: A network approach, European Journal of Political Research, 36(257-281.

Pollack, M. A. (1994). Creeping Competence: The Expanding Agenda of the European Community, Journal of Public Policy, 14(2), 95-145.

Radaelli, C. (1999). Technocracy in the European Union: Political Dynamics of the European Union. London: Longman.

Scharpf, F. W. (1999). Governing in Europe: Effective and democratic? Oxford: Oxford University Press.

Scharpf, F. W. (2012). Legitimacy Intermediation in the Multilevel European Polity and Its Collapse in the Euro Crisis, MPIfG Discussion Paper 12(6).

Schrage, D. (2012). Flüssige Technokratie, MERKUR, Heft 09/10(September 2012), 817-825.

Siefken, S. T. (2007). Expertenkommissionen im politischen Prozess: eine Bilanz zur rot-grünen Bundesregierung 1998 - 2005. Wiesbaden: VS Verlag für Sozialwissenschaften.

Streeck, W. (2012). Wissen als Macht, Macht als Wissen. Kapitalversteher im Krisenkapitalismus, MERKUR, Heft 09/10(September 2012), 776-787.

Tsakatika, M. (2005). Claims to Legitimacy: The European Commission between Continuity and Change, JCMS: Journal of Common Market Studies, 43(1), 193-220.

Vos, E. (1999). EU Committees: the Evolution of Unforeseen Institutional Actors in European Product Regulation, In: Joerges, C.Vos, E. (Hrsg.), EU Committees: Social Regulation, Law and Politics. Oxford / Portland: Hart Publishing, 19-47.

Walter, F. (2012). Zivilgesellschaft und Partizipationsdemokratie. Ein Albtraum?, INDES - Zeitschrift für Politik und Gesellschaft, 2012(4), $135-143$

Weingart, P. \& Lentsch, J. (2008). Wissen - Beraten - Entscheiden. Form und Funktion wissenschaftlicher Politikberatung in Deutschland. Weilerwist: Verbrück.

Zegart, A. B. (2004). Blue Ribbons, Black Boxes: Toward a Better Understanding of Presidential Commissions, Presidential Studies Quarterly, 34(2), 366-393.

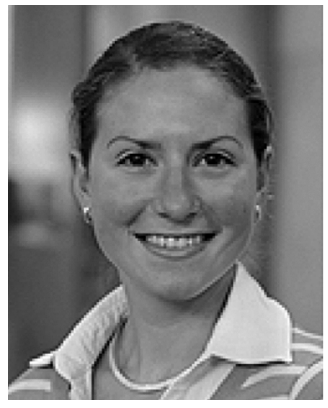

Dr. Julia Metz ist wissenschaftliche Mitarbeiterin der Schumpeter Nachwuchsgruppe „Positionsbildung in der EU-Kommission“ am WZB. Sie studierte Politikwissenschaft und VWL in Heidelberg und Stockholm und wurde 2012 an der Freien Universität Berlin promoviert. Email: julia.metz@wzb.eu 